\title{
BetterGeoEdu: el uso de Minecraft como instrumento de aprendizaje de la importancia de las materias primas y la economía circular entre alumnos de Educación Primaria BetterGeoEdu: the use of Minecraft as a learning tool on the importance of raw materials and the circular economy among Primary Education students
}

\author{
Juan Herrera Herbert ${ }^{1}$, Jorge Luis Costafreda Mustelier ${ }^{1}$, Christian Peña Narciso ${ }^{1}$, Domingo Alfonso Martín Sánchez ${ }^{1}$ \\ juan.herrera@upm.es; jorgeluis.costafreda@upm.es; christianpn@alumnos.upm.es; domingoalfonso.martin@upm.es \\ ${ }^{1}$ Departamento de Ingeniería Geológica y Minera (Department of Mining and Geological Engineering) \\ Universidad Politécnica de Madrid (Technical University of Madrid) \\ Escuela Técnica Superior de Ingenieros de Minas y Energía (Higher Technical School of Mining and Energy Engineering) \\ Madrid, ESPAÑA (SPAIN)
}

\begin{abstract}
Resumen- El uso de juegos como herramientas de aprendizaje se ha popularizado como forma de aprender mientras los estudiantes se divierten. En el ámbito del proyecto BetterGeoEdu, se ha creado BetterGeo, que es una modificación (un mod) de distribución gratuita para Minecraft, uno de los juegos más populares del mundo, destinada a mejorar y ayudar a los estudiantes, especialmente de primaria y secundaria, a familiarizarse con la importancia de las materias primas en la vida cotidiana mientras profundizan en conceptos básicos sobre geología, minerales, minería, procesamiento de minerales y economía circular. Junto con la implementación del mod, el proyecto BetterGeoEdu también desarrolla de materiales de aprendizaje para ayudar a profesores y estudiantes en este acercamiento al sector de las materias primas. BetterGeoEdu es un proyecto europeo financiado por EIT Raw Materials, una de las ocho Comunidades de Conocimiento e Innovación (KICs) iniciadas por el EIT (Instituto Europeo de Innovación y Tecnología) que es un órgano de la Comisión Europea. En este trabajo se describe el Proyecto BetterGeoEdu, su alcance y sus objetivos junto con el desarrollo innovador de ejercicios y material educativo de distribución gratuita diseñado destinado a facilitar a los alumnos ese primer contacto con las materias primas.
\end{abstract}

Palabras clave: Juegos, aprendizaje interactivo, materias primas, cemento, economía circular, desarrollo estudiantil, enseñanza innovadora

\footnotetext{
Abstract- The use of games as learning tools has become popular as a way to learn while students are having fun. In the scope of the BetterGeoEdu project, BetterGeo has been created, which is a free distribution modification (a mod) for Minecraft, one of the most popular games in the world, aimed at improving and helping students, especially from primary and secondary schools, to get acquainted with the importance of raw materials in everyday life while delving into the basics of geology, minerals, mining, mineral processing, and the circular economy. Along with the implementation of the mod, the BetterGeoEdu project also develops learning materials to help teachers and students in this approach to the raw materials sector. BetterGeoEdu is a European project funded by EIT Raw Materials, one of the eight Knowledge and Innovation Communities (KICs) initiated by the EIT (European Institute of Innovation and Technology) which is a body of the European Commission. This paper describes the BetterGeoEdu
}

Project, its scope and its objectives, together with the innovative development of exercises and educational material for free distribution designed to facilitate that first contact with raw materials for students.

Keywords: Gaming, interactive learning, raw materials, cement, circular economy, student development, innovative teaching

\section{INTRODUCCIÓN}

En los últimos años y, quizás de una forma más acentuada como consecuencia de la pandemia de SARS-CoV-2 (COVID19), se ha producido una evolución muy destacada en las técnicas y metodologías de enseñanza que, aprovechando el potencial de la informática y la extensión y dimensión de los juegos informáticos, ha dado lugar a la creación y rápida propagación de nuevos sistemas para transmitir la educación (D.A. Martín et al, 2017). Estos sistemas se desarrollan en un escenario interactivo, no presencial, pero igualmente eficaz, cómodo y simultáneo. El avance de la pandemia mundial, la extensión y duración de los confinamientos sanitarios y el crecimiento e implantación de la "enseñanza a distancia" además de toda clase de actividades on-line, ha dado lugar a un crecimiento aun mayor del esperado de iniciativas como el proyecto BetterGeoEdu (P. Westrin, 2020).

En éste contexto de uso de juegos como herramientas de aprendizaje en el que los alumnos de distintas edades son capaces de aprender múltiples cuestiones y desarrollar competencias y habilidades mientras se divierten es donde se encuadra el Proyecto BetterGeoEdu (https://www.bettergeoedu.com), que ha desarrollado una modificación (un mod) del juego del Minecraft, uno de los juegos más populares del mundo, para mejorar ayudar a los alumnos de primaria a familiarizarse con la importancia de las materias primas en la vida moderna y empezar a aprender conceptos básicos sobre geología, minerales, minería, procesamiento de minerales y economía circular. 
Este proyecto está siendo desarrollado por un consorcio coordinado por Geological Survey of Sweden, SGU (Suecia), y constituido, además, por la Universidad Politécnica de Madrid - UPM (España), el Consiglio Nazionale delle Ricerche - CNR (Italia), Geological Survey of Slovenia - GeoZS (Eslovenia), Montanuniversität Leoben (Austria), Tallinn University of Technology (Estonia), Trinity College Dublin (Irlanda), Université de Liège (Bélgica), Universidad de Limerick (Irlanda), Turvallisuus- ja kemikaalivirasto - Tukes (Finlandia). El proyecto está financiado por el EIT Raw Materials. EIT Raw Materials es una de las ocho Comunidades de Conocimiento e Innovación creadas para impulsar la innovación y el emprendimiento en toda Europa con el apoyo del Instituto Europeo de Innovación y Tecnología (EIT).

En esta publicación se describen la idea en la que se basa BetterGeo, los objetivos y los socios del consorcio, así como el desarrollo innovador de ejercicios y material educativo diseñado para facilitar a los alumnos de primaria el primer contacto con las materias primas.

\section{CONTEXTO}

El Proyecto BetterGeoEdu surgió siguiendo la idea de que los juegos son herramientas muy útiles y eficaces como instrumento para potenciar el aprendizaje. Aprender divirtiéndose ayuda a los estudiantes a retener mejor los conocimientos porque el proceso es más agradable y es muy fácil memorizar ideas y conceptos que, en otras circunstancias, sería mucho más difícil. Por otro lado, el juego de Minecraft, que es uno de los más populares del mundo, con millones de descargas al día, ha conseguido una enorme difusión a nivel mundial, con millones de jugadores. Fruto de la unión, surge la utilización de Minecraft como una herramienta muy útil y de un enorme potencial para el aprendizaje por todos de la importancia de las materias primas en la vida moderna y empezar a aprender conceptos básicos sobre materias primas (geología, minerales, minería, procesamiento de minerales), economía circular sostenibilidad y mucho más.

El proyecto ha sido fuertemente apoyado y financiado por la Unión Europea, a través del EIT Raw Materials, porque Europa está comprometida con el desarrollo y el uso sostenible de las materias primas. El documento "The European Green Deal" la hoja de ruta de un plan de acción completo y concreto para impulsar el uso eficiente de los recursos en una economía limpia y circular, que restaure la biodiversidad y, sobre todo, reduzca la contaminación. Para que Europa pueda ser más competitiva al tiempo que se hace más ecológica y circular, la industria necesitará un suministro seguro de energía y materias primas limpias y asequibles. Garantizar el suministro de materias primas sostenibles mediante la diversificación del abastecimiento a partir de fuentes primarias y secundarias es, por tanto, uno de los requisitos previos para hacer realidad esta transición. Esto implica inculcar a la sociedad el conocimiento de los recursos disponibles en el territorio europeo y en el resto del mundo, para qué se utilizan y qué productos de la vida cotidiana existen gracias a ellos, así como inculcar también la certeza de que su existencia es finita y limitada, por lo que la explotación de los mismos debe ser responsable.

EIT Raw Materials es una de las ocho Comunidades de Conocimiento e Innovación creadas para impulsar la innovación y el emprendimiento en toda Europa. Creada en
2015 y participada por más de 120 socios europeos de las principales industrias, universidades e instituciones de investigación de más de 20 países de la UE, EIT Raw Materials es el mayor consorcio del sector de las materias primas a nivel mundial. Sus socios actúan en toda la cadena de valor de las materias primas, desde la exploración sostenible, la minería eficiente y el procesamiento de minerales hasta la sustitución, el reciclaje y la economía circular. Tiene la visión de convertir las materias primas en una fuerza importante para Europa mediante la búsqueda de soluciones nuevas e innovadoras para asegurar el suministro y mejorar el sector de las materias primas en Europa, y la misión de contribuir a impulsar la competitividad, el crecimiento y el atractivo del sector europeo de las materias primas a través de la innovación radical, los nuevos enfoques educativos y el espíritu empresarial.

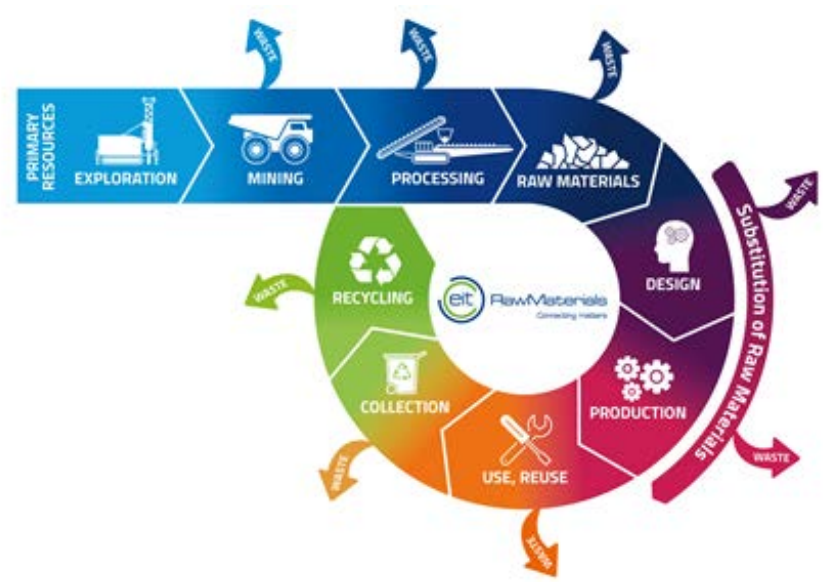

Figura 1. El abastecimiento de materias primas en una economía circular. (https://eitrawmaterials.eu, 2018)

La EIT Raw Materials Academy es la rama que engloba todas las actividades educativas del EIT Raw Materials. Las actividades de todo el ecosistema de alumnos (estudiantes de doctorado, estudiantes de máster, socios industriales, profesionales del sector de las materias primas y la sociedad en general) fomentan nuevas formas de aprendizaje y enseñanza al conectar el mundo académico, la industria y las organizaciones de investigación. El objetivo es formar a personas que tendrán una mentalidad abierta, que conozcan toda la cadena de valor de las materias primas y sean capaces de desarrollar sus funciones en nuevos entornos de trabajo, fomentando las habilidades, conocimientos y actitudes emprendedoras y de innovación necesarias para los emprendedores e intraemprendedores del mañana.

\section{DESCRIPCIÓN}

BetterGeo es una modificación (un mod) para Minecraft creada por el Servicio Geológico de Suecia (SGU) para introducir en el juego nuevas características y funcionalidades que mejoras las prestaciones desde el punto de vista del aprendizaje de la geología y la minería. El proyecto también incluye la preparación de múltiples ejercicios y actividades complementarias que se pueden hacer durante el juego o separadamente, y tanto en casa como en el colegio, en el ámbito de actividades complementarias en el aula. El mod de BetterGeo aporta una geología más realista al juego, con rocas adicionales, suelos, materias primas, herramientas, objetos y 
muchos más elementos. El mod enseña acerca de las materias primas y sobre su importancia en la sociedad moderna. Además, el mod ofrece nuevas oportunidades al jugador gracias a bloques y características más realistas, así como nuevos objetos de la vida cotidiana para fabricar. Además, BetterGeo está disponible para su descarga gratuita, sin ningún coste para el usuario que tenga el juego.

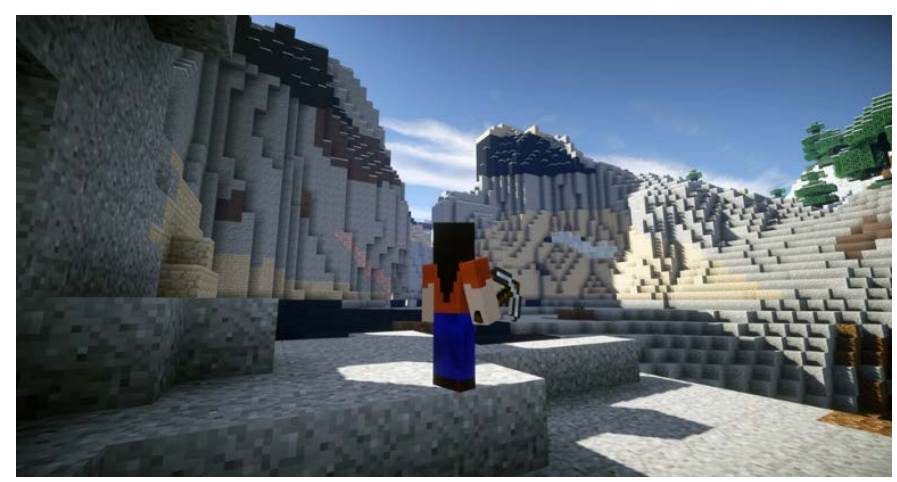

Figura 2. Imagen del juego. (https://www.bettergeoedu.com, 2020)

BetterGeo incorpora diferentes ejercicios y una amplia variedad de material educativo adicional, traducidos a todos los idiomas, para utilizar en el aula, que vienen complementados con instrucciones para el profesor, consejos y trucos para su mejor aprovechamiento, pero especialmente, dando la capacidad a los docentes de particularizarlos y enfocarlos a las distintas dinámicas que cada profesor quiera diseñar con sus alumnos. En este sentido, es una herramienta muy versátil que se alinea con la creatividad del docente para crear situaciones atractivas e interesantes a los alumnos. El hecho de que puede utilizarse tanto en el aula como en casa, también facilita la integración de los padres para conseguir dinámicas familiares. Un aspecto importante a destacar es que hay ejercicios que no requieren la utilización de un ordenador ni de una conexión a internet, o bien que pueden desarrollarse con o sin ordenador, lo que incrementa el universo de posibilidades.

Todos los ejercicios han sido desarrollados por los miembros del consorcio y están preparados y dirigidos principalmente a ser utilizados por estudiantes de educación primaria, pero pueden ser utilizados por personas de todas las edades. De hecho, pueden ser utilizados de manera provechosa por estudiantes universitarios de Ingeniería o de Ciencias de la Tierra. Precisamente es el caso de los ejercicios de reconocimiento de minerales y rocas que aquí se describen.

Este ejercicio ha sido desarrollado por el equipo de la Universidad Politécnica de Madrid y permite a los alumnos y jugadores aprender y coger destrezas en el procedimiento que se debe seguir para la descripción y reconocimiento de rocas y minerales, incluso sin tener conocimientos previos sobre estos materiales. Al mismo tiempo, se pretende que desarrollen la capacidad de deducir, de forma intuitiva, su uso y sus aplicaciones en diferentes ámbitos de la sociedad actual.

El procedimiento tiene dos modalidades: a) reconocimiento de minerales y b) reconocimiento de rocas. Las muestras de minerales y rocas aparecen en cajas de muestras reales que se distribuyen entre los colegios, o bien virtuales, cuando los jugadores no tienen físicamente acceso a estas cajas de muestras (Figura 3).

Las características fundamentales que definen a los minerales son el color, la forma, el brillo, la dureza, la transparencia, la fragilidad, la maleabilidad, la ductilidad, la elasticidad y el magnetismo, entre otras propiedades (L. Robb, 2004). También se deben conocer las condiciones naturales en las que se formaron los minerales y las rocas, considerando factores como la presión y la temperatura (Tarbuck et all, 2008). Con este ejercicio, el alumno trabajará con algunas de las propiedades básicas de los minerales y las rocas antes mencionadas.

\section{A. Metodología para el reconocimiento de minerales de forma interactiva}

En esta parte del ejercicio, el alumno hace clic en la caja de muestras de minerales. Con este gesto, la caja virtual se abre en la pantalla y aparecen varias muestras de minerales. El alumno puede entonces elegir libremente cualquiera de las muestras de minerales dispuestas en la caja; Por ejemplo, supongamos que el alumno hace clic en la muestra 1.

Con esta acción, aparece en pantalla una imagen de la muestra (como se observa en la Figura 3), junto con una serie de comandos e instrucciones específicas que el alumno debe seguir. Cada instrucción es una actividad a desarrollar:

Actividad 1: "Selecciona la muestra haciendo clic sobre ella"

Actividad 2: "¿De qué color es este mineral?" Describe el color y las tonalidades del mineral. Si la descripción es correcta, aparece un comando que ofrece al usuario más información sobre los colores y matices. Si la información introducida es errónea, el alumno debe volver a empezar. El alumno no puede acceder a la actividad 3 hasta que no haya completado la actividad 2 .

Actividad 3: "¿Qué forma tiene este mineral?" La orden indica al alumno que describa la forma del mineral, es decir, si tiene geometría, si tiene caras bien definidas o si es irregular. La respuesta correcta activa una nueva opción, en la que el alumno puede ampliar una subpantalla en la que puede conocer información adicional sobre la actividad realizada. Una respuesta incorrecta obliga al alumno a volver a empezar. El acceso a la actividad 4 no está permitido hasta que se haya completado la actividad 3.

Actividad 4: "¿Qué brillo tiene este mineral?". En esta actividad se pide al alumno que describa el brillo del mineral. Debe responder si es metálico, como los metales, o si por el contrario, es no metálico, como la mayoría de los objetos que no son metálicos. La respuesta correcta abre un camino que proporciona al alumno una explicación ampliada sobre el brillo de la muestra. La respuesta incorrecta obliga al alumno a retroceder de nuevo. Una vez más, la actividad de acceso 5 no aparecerá hasta que se haya completado la actividad 4.

Actividad 5: ¿Crees que este mineral es duro? El comando plantea una pregunta al alumno sobre la dureza del mineral. Una respuesta correcta da la posibilidad de ampliar el conocimiento sobre la dureza del mineral, comparándolo con minerales más o menos duros que la muestra en estudio. Si el alumno elige la opción incorrecta, tiene que volver a empezar. No se puede 
acceder a la actividad 6 hasta que se haya completado la actividad 5.
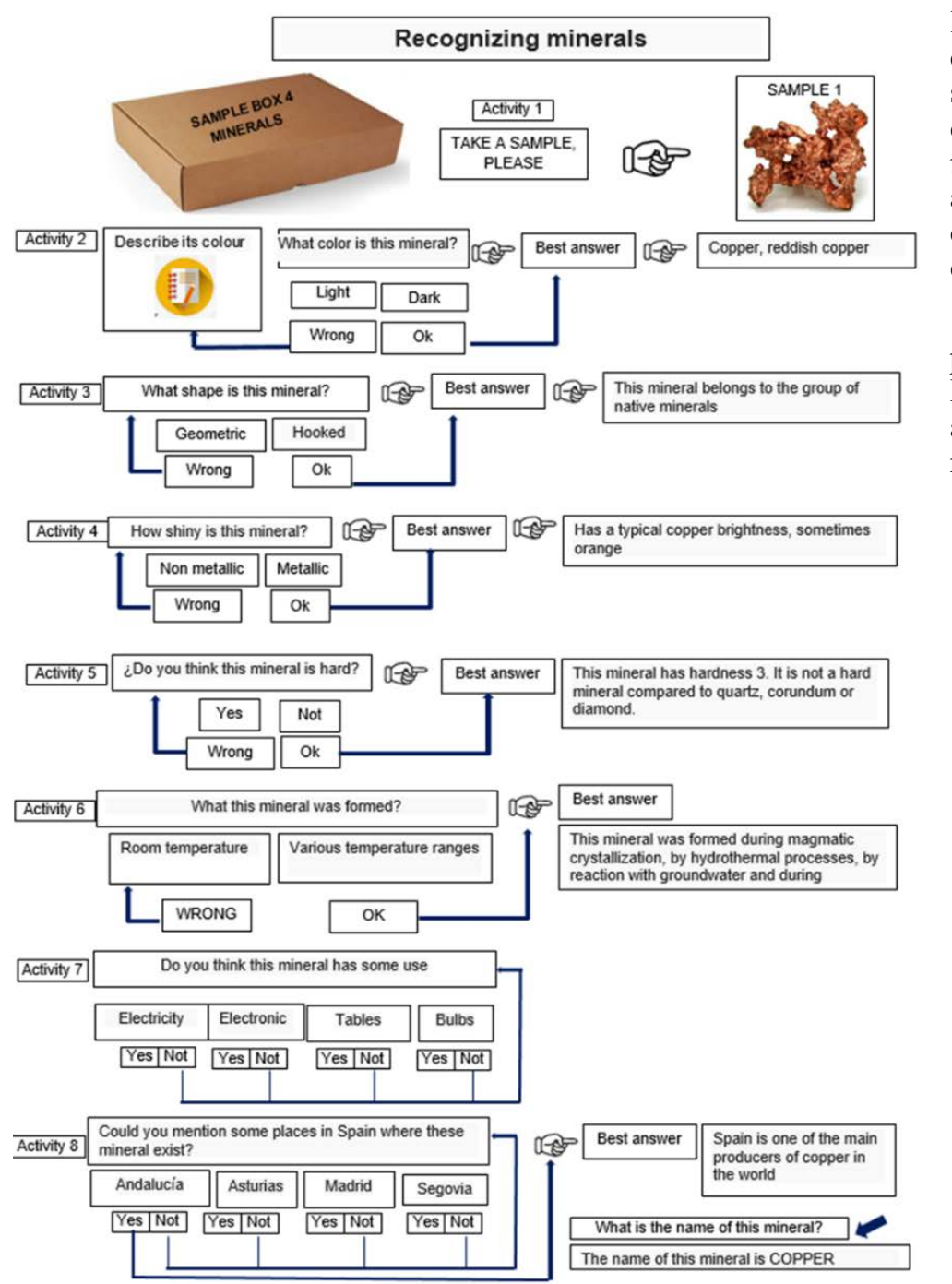

Figura 3. Metodología para el reconocimiento interactivo de minerales. Esquema propuesto para el proceso de aprendizaje de reconocimiento de minerales para alumnos de primaria.

Actividad 6: "¿Cómo se formó este mineral?" En esta actividad, el alumno elige una de las respuestas a la pregunta de cómo se formó el mineral en la naturaleza: a baja, media o alta temperatura. La respuesta adecuada activa una opción con más explicaciones al respecto. La respuesta incorrecta obliga al alumno a volver al principio de esta actividad. Al igual que en las actividades anteriores, el acceso a la actividad 7 no se abrirá hasta que se haya completado con éxito la actividad 6.

Actividad 7: ¿Crees que este mineral tiene alguna utilidad? En esta actividad se pregunta al alumno sobre el uso del mineral estudiado. En la pantalla aparecen varios comandos (cuatro en total), y cada uno tiene dos subcomandos adicionales que muestran al alumno posibilidades correctas o incorrectas. Si el alumno elige al menos dos respuestas incorrectas, volverá al principio de la actividad 7. Si todas las respuestas son correctas, se abre el acceso a la actividad 8 .
Actividad 8: "¿Puedes mencionar algún lugar de España donde se pueda encontrar este mineral?" Con esta actividad, el alumno debe aprender dónde se encuentran los yacimientos del mineral estudiado en España (para otros países, se prepararán las versiones correspondientes). En la pantalla se abren cuatro comandos que indican cuatro regiones. Dos de esas regiones son erróneas y dos son correctas. La elección de las regiones erróneas obliga a comenzar de nuevo la actividad 8. Las respuestas correctas permiten acceder a una pantalla donde el alumno puede obtener información adicional y ampliar sus conocimientos. Simultáneamente, el alumno accede a un espacio donde se muestra el nombre del mineral.

Una vez completadas todas las actividades, el alumno puede pulsar el comando "QUIERO SABER MÁS SOBRE EL USO DE ESTE MINERAL". Esta opción permite al alumno acceder a los diferentes usos y aplicaciones de este mineral a través de imágenes (ver figura 4).

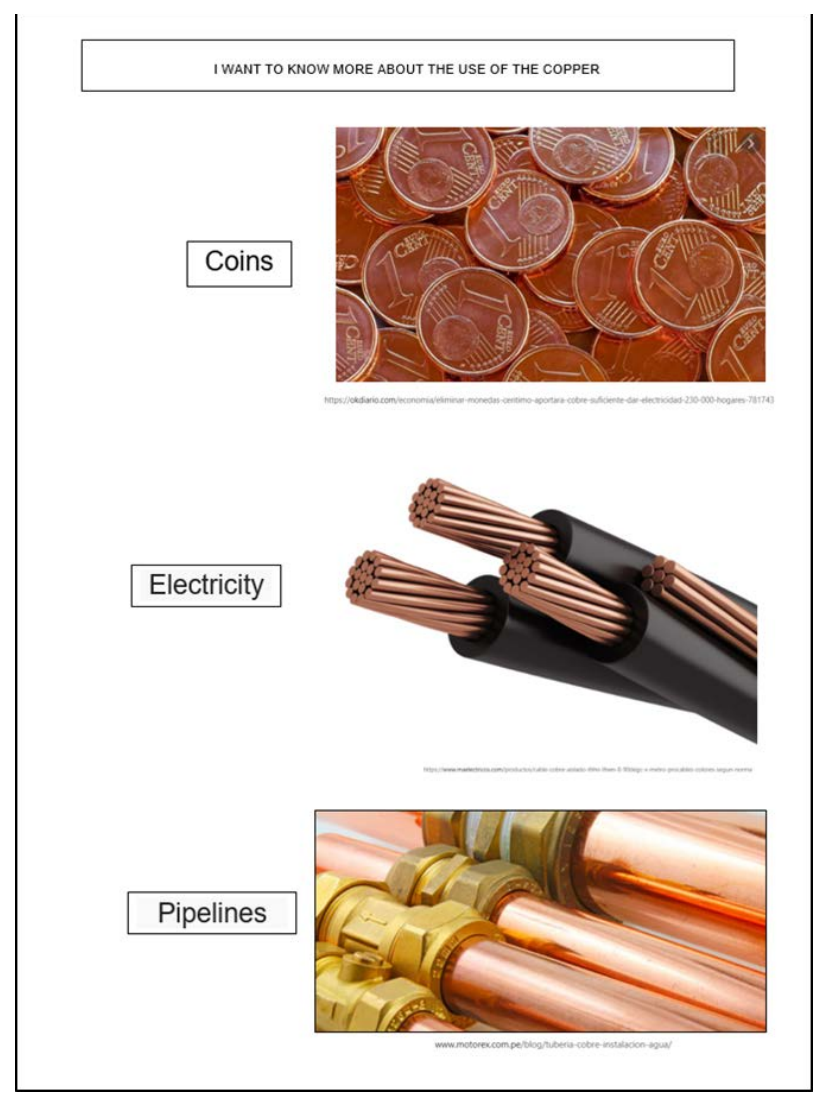

Figura 4. Ejemplos gráficos del uso y las aplicaciones de los minerales.

\section{$B$. Metodología para el reconocimiento de rocas}

Las características fundamentales que definen a las rocas son el color, la cristalinidad, la dureza, la textura y la estructura, entre otras propiedades. Al igual que los minerales, hay que determinar las condiciones de formación, la presión, la temperatura y las alteraciones que las afectan. A través de este ejercicio se pretende que el alumno sea capaz de distinguir entre minerales y rocas, mediante preguntas muy sencillas. El ejercicio termina con la identificación del nombre de la roca. 
El alumno hace clic en el cuadro de muestras de rocas. En la pantalla se abrirá la caja y aparecerán diferentes muestras de rocas. El alumno debe pulsar libremente sobre cualquier muestra de roca, lo que mostrará la imagen de la muestra, así como los comandos disponibles para el desarrollo de las actividades (ver figura 5).

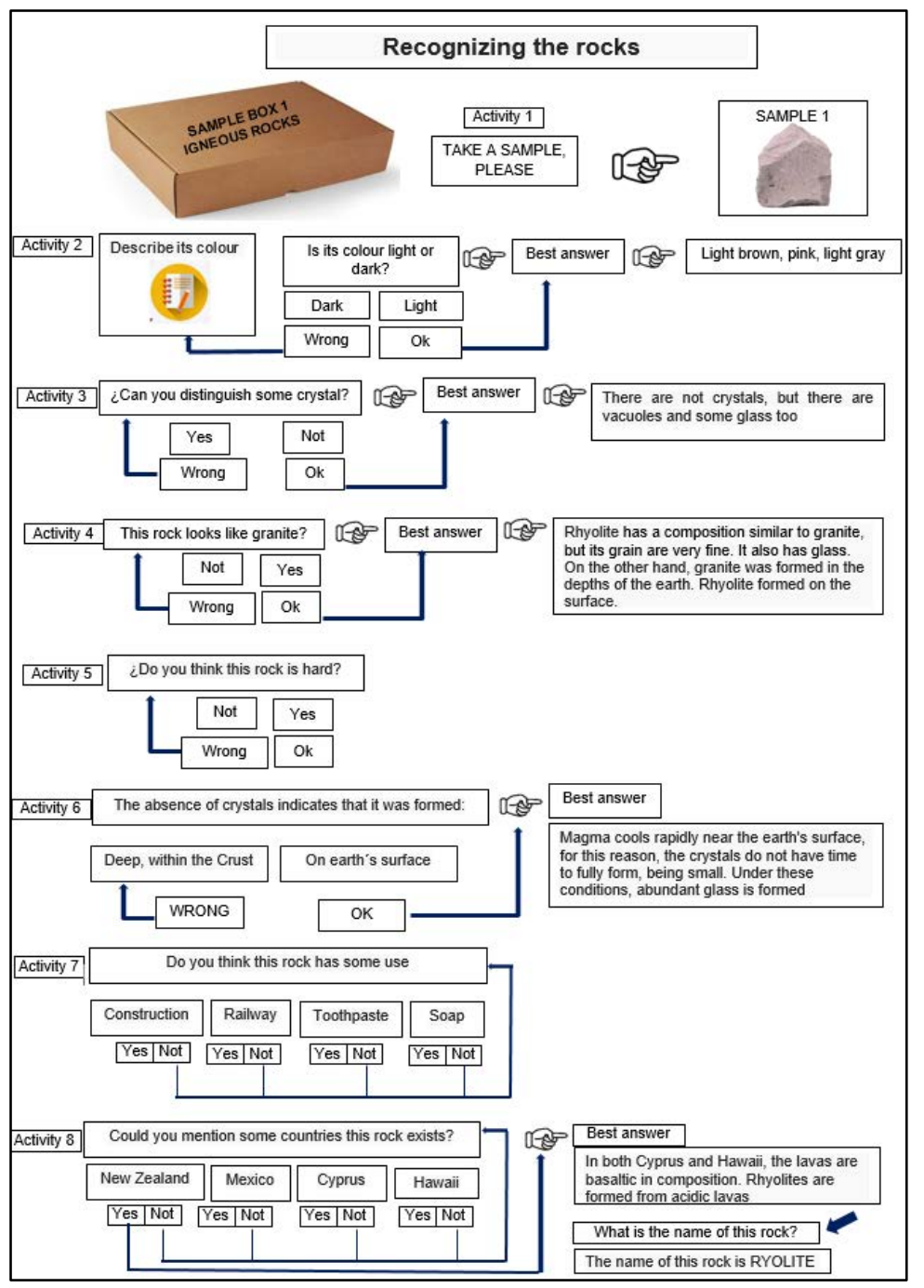

Figura 5. Diagrama propuesto para el proceso de aprendizaje de reconocimiento de rocas para alumnos de primaria.

Actividad 1: "Selecciona la muestra haciendo clic sobre ella"

Actividad 2: Descripción del color: "¿El color es claro u oscuro? La descripción correcta activa un cuadro que proporciona más información sobre los colores y los tonos. Si la respuesta es incorrecta, el alumno debe volver a empezar. No se puede acceder a la actividad 3 hasta que se haya completado la actividad 2.

Actividad 3: Esta actividad plantea la pregunta: ¿¿Puedes distinguir algún cristal?". El alumno elegirá la opción que considere correcta. La opción correcta muestra una nueva ventana con información adicional sobre la cristalinidad de la roca. La respuesta incorrecta le hará retroceder. El alumno no puede acceder a la actividad 4 hasta que no haya completado la actividad 3.
Actividad 4: En esta actividad se pregunta al alumno: "¿Se parece esta roca al granito?" El granito es una roca muy común, y la mayoría de los estudios sobre rocas se basan en la comparación con el granito. La respuesta correcta permite al alumno acceder a una explicación más extensa de esta pregunta. La respuesta incorrecta devuelve al alumno al principio de esta actividad. No se puede acceder a la actividad 5 hasta que se haya completado la actividad 4.

Actividad 5: En esta actividad se pregunta sobre la dureza de la roca: "¿Crees que esta roca es dura?". La elección errónea conduce al inicio de esta actividad. El alumno no puede acceder a la actividad 6 hasta que no haya completado correctamente la actividad 5.

Actividad 6: En esta actividad, el alumno debe responder a la siguiente pregunta: "La ausencia de cristales indica que esta roca se formó En las profundidades, dentro de la corteza o en la superficie de la Tierra". Si el alumno responde correctamente, encuentra una opción con más explicaciones sobre las cuestiones planteadas. La respuesta incorrecta obliga al alumno a volver al principio de esta actividad. El alumno no puede acceder a la actividad 7 hasta que no haya completado satisfactoriamente la actividad 6 .

Actividad 7: En esta actividad se pregunta al alumno: "¿Crees que esta roca tiene alguna utilidad?". Como en el caso de los minerales, hay cuatro comandos con diferentes opciones, y cada comando tiene dos subcomandos adicionales con respuestas correctas e incorrectas. Elegir al menos una opción incorrecta envía al alumno al primer paso de esta actividad. Las respuestas correctas dan acceso a la actividad 8 .

Actividad 8: En esta actividad se pregunta al alumno dónde se pueden encontrar los yacimientos de este tipo de roca, a través de la siguiente pregunta: "¿Podrías mencionar algunos países donde existe esta roca?". Se activan cuatro comandos que indican cuatro países diferentes. Dos de estos países son erróneos y los otros dos son correctos. La elección de los países erróneos envía al alumno al principio de la actividad. Las respuestas correctas dan acceso a una pantalla con más información. Al mismo tiempo, el alumno puede acceder a una zona donde puede identificar el nombre de la roca.

A continuación, el alumno puede hacer clic en un comando que tiene la siguiente etiqueta "QUIERO SABER MÁS SOBRE EL USO DE ESTA ROCA", donde puede aprender, a través de ejemplos de imágenes, los usos y aplicaciones de la roca estudiada (ver figura 6).

\section{Resultados}

Aunque el desarrollo de estos ejercicios está en las fases iniciales de implantación y difusión, el logro más importante de esta herramienta es que el alumno gestiona todo el proceso por sí mismo. Al tratarse de una herramienta educativa complementaria de fácil uso para los profesores, se espera una acogida favorable en el ámbito de las actividades escolares complementarias o incluso extraescolares.

Figura 6. Ejemplos gráficos del uso y las aplicaciones de las rocas.

Como se ha indicado, estos ejercicios son perfectamente utilizables por alumnos universitarios y es por ello que se está 


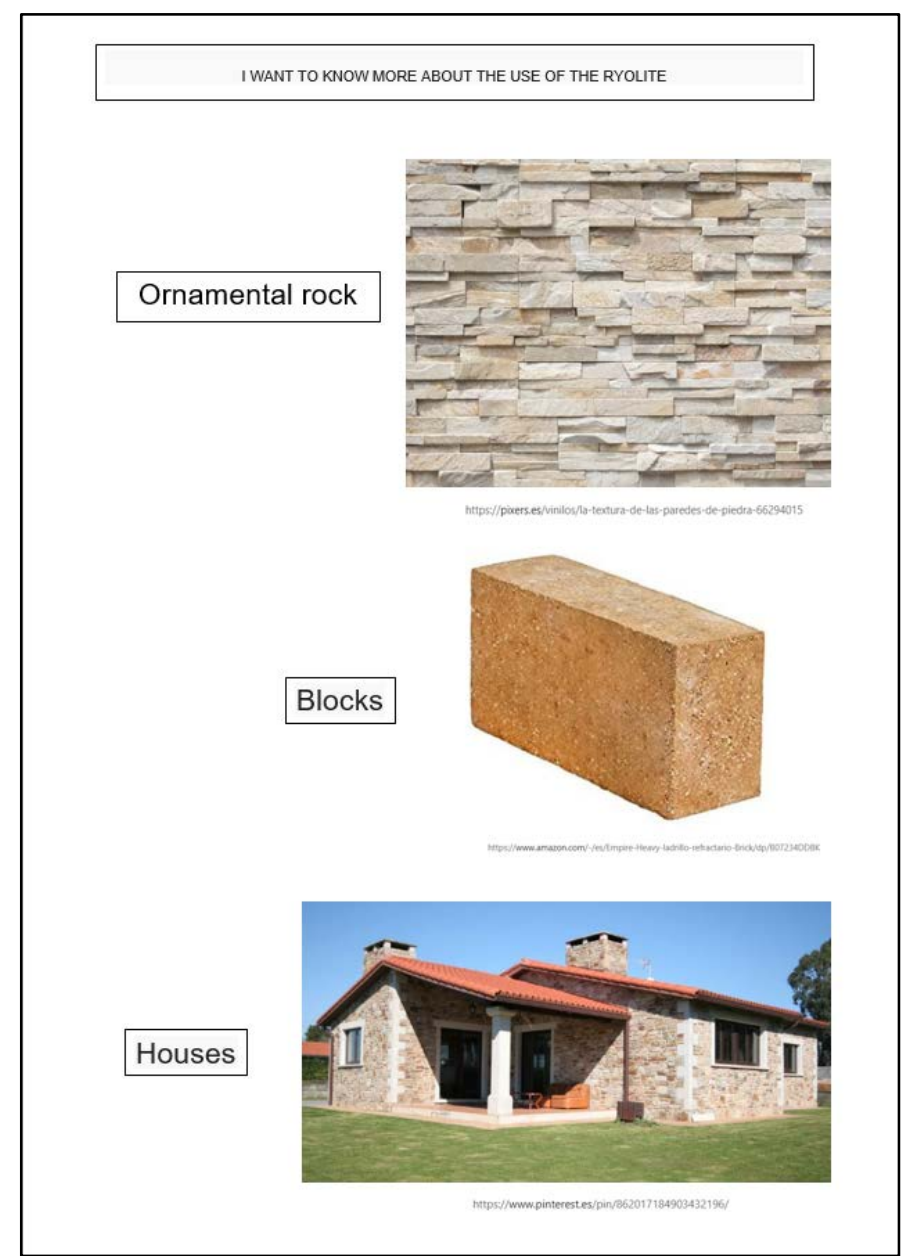

diseñando un sistema de evaluación del impacto a través de los alumnos de prácticas de las asignaturas de geología en las titulaciones de Grado en Ingeniería en Tecnologías Mineras (GITM), Grado en Ingeniería Geológica (GIG) y Grado en Ingeniería en Tecnologías Ambientales (GITA) de la UPM. Se utilizará un grupo patrón de notas de acceso similar y se verá la mejora en los resultados. Además, se propondrá un equipo de testeo en titulaciones no relacionadas con asignaturas de Geología como puede ser la titulación de Grado de Ingeniero de la Energía (GIE).

Además, se elaborará una encuesta en la que dichos estudiantes valoraran pros y contras del programa. A partir de aquí podrán elaborar estadísticas que permitan valorar el índice de penetración de esta metodología de enseñanza basada en Gamificación.

\section{CONCLUSIONES}

El juego propuesto como método educativo, compuesto por diferentes ejercicios y actividades, permite que los alumnos de primaria puedan empezar a reconocer tantas variedades de minerales y rocas como deseen. Además, tiene la ventaja de que se puede acceder a él desde cualquier soporte informático y desde cualquier lugar, y además es gratuito. Es un sistema de aprendizaje innovador, rápido, cómodo, interactivo y eficaz.

El método puede ajustarse a diferentes niveles de complejidad, según la edad del alumno; es decir, puede dirigirse a estudiantes desde la enseñanza primaria hasta la universitaria.
Si el grado de penetración del juego es suficientemente importante se podría pensar en su implantación en la docencia practica en entornos de no presencialidad. Otra posible aplicación es el refuerzo en la adquisición de competencias de estudiante que no superan la convocatoria ordinaria y deciden mantener y reforzar, con ayuda de este juego, el nivel de conocimiento que no les hizo apto para supera las pruebas de nivelación de las asignaturas de geología.

\section{REFERENCIAS}

BetterGeo (2018) "Teach about raw materials with Minecraft!". Retrieved from https:/www.bettergeoedu.com/

European Institute of Innovation \& Technology. EIT - Making innovation happen. Accessed 28 December 2018. Retrieved from https:// eit.europa.eu

EIT Raw Materials. Developing Raw Materials into a major strength for Europe. Accessed 28 December 2018. Retrieved from https:// eit.europa.eu/eit-community/eitraw-materials

Escribano, M.; López Jimeno, C. Mataix, C. (2019). Manual de minerales críticos y estratégicos en la nueva economía. Grupo de Proyectos de Ingeniería. ISBN: 978-84-96140$62-2$.

European Commission. (2019). "The European Green Deal". Retrieved from https://ec.europa.eu/

García Laso, A. (2014). "Development of competencies in engineering education through participation in programs with social organizations. The case of the Unit of Social Entrepreneurship, Ethics and Values in Engineering". (Doctoral dissertation), Universidad Politécnica de Madrid - Technical University of Madrid.

Martín, D. A., \& García-Laso, A. (2014). "Experiences in social innovation: a platform for ethics through a school of engineering studies". Journal of Cases on Information Technology, 16(3), 4-17. doi:10.4018/jcit.2014070102

Martín, D.A.; García, A.; Costafreda Mustelier J.L. (2019). "Challenges in educational innovation: eco-social values in engineering education". Sistemas ; pp. 1-3. ISSN 02100223.

Robb, L. (2004). "Introduction to ore-forming processes". Ed. Blackwell Publishing. ISBN: 0-632-06378-5. 386 págs.

Sánchez, M. E.; Hernández, Y.; Hernández S. (2019). "Teaching innovation in human resources through service-learning. A pilot experience”. RIDAS. Revista Iberoamericana de Aprendizaje Servicio, 7, 1-16.

Service-Learning. (n.d.). Service-Learning. Teaching \& learning innovation. Retrieved from https://servicelearning.utk.edu/1705-2/

Tarbuck, E. J.; Lutgens, F. K. (2008). "Earth sciences. An Introduction to Physical Geology". $8^{\mathrm{a}}$ edición. Published by Pearson Education, Inc, publishing as Prentice Hall, Copyright (C) 2005. Pages.712. ISBN: 0-13-114865-6

Westrin, P. (2020) "Educational material about raw materials, circular economy \& sustainability supported by BetterGeoEdu". https://www.bettergeoedu.com/ 